\title{
Sarcoma de Kaposi ganglionar postrasplante renal: reporte de dos casos en un centro de trasplante colombiano
}

\section{Kaposi sarcoma in lymph nodes after kidney transplantation: report of two cases in a Colombian transplant center}

\author{
Dabely A. Palacios-Ramírez', Gabriel Jaime Varela-Aguirre²
}

Resumen. El cáncer es una causa importante de morbilidad y mortalidad en los receptores de trasplante. La combinación de infecciones virales, terapia de inmunosupresión y la alteración en el sistema inmune en los pacientes trasplantados, contribuyen al desarrollo de cáncer. El sarcoma de Kaposi es causado por el virus herpes humano 8 (VHH-8), y aunque es raro en la población general, puede ser hasta 300 veces más frecuente en los pacientes con trasplante renal. El diagnóstico de la enfermedad se realiza a menudo con base en las características de las lesiones, pero debe ser confirmado por histología. En años recientes, los inhibidores de mTOR han mostrado ser efectivos para el control del sarcoma de Kaposi en los pacientes trasplantados, ya que se interrumpe el efecto antiapoptótico y la angiogénesis dependientes de la proteína mTOR, los cuales son esenciales para el desarrollo y la propagación de células malignas. Se presentan dos casos de pacientes con sarcoma de Kaposi ganglionar, sin lesiones en piel, en nuestro centro de trasplante, quienes respondieron de manera positiva al cambio del esquema inmunosupresor con inhibidores de mTOR.

Palabras clave: sarcoma de Kaposi, herpesvirus humano 8, trasplante de riñón, $m T O R$, serina-treonina quinasas $m T O R$, ganglios linfáticos.

Abstract. Cancer is a major cause of morbidity and mortality in transplant recipients. The combination of viral infections, immunosuppression therapy and immune system dysfunction in transplant patients contribute to the development of cancer. Kaposi sarcoma is caused by human herpes virus 8 (HHV-8) and although rare in the general population,

\footnotetext{
${ }^{1}$ Médica, Especialista en Medicina Interna y Nefrología. Grupo de Trasplantes, Hospital Universitario San Vicente Fundación. Rionegro, Colombia. E-mail: dabelypalacio@yahoo.com.

${ }^{2}$ Médico, Especialista en Patología, Dermatopatología y Patología Oncológica. Departamento de Patología, Hospital Universitario San Vicente Fundación. Rionegro, Colombia. Hospital Pablo Tobón Uribe, Clínica Aurora Centro Especializado en Cáncer de Piel. Medellín, Colombia.

Conflicto de interés: los autores declaran que no tienen conflicto de interés.

Medicina \& Laboratorio 2021;25:525-534. https://doi.org/10.36384/01232576.437.

Recibido el 22 de diciembre de 2020; aceptado el 23 marzo de 2021. Editora Médica Colombiana S.A., 2021
} 
it is reported to be up to 300 times more common in kidney transplant patients. Diagnosis of the disease is often made on the basis of the characteristic appearance of lesions, but must be confirmed by histology. In recent years, mTOR inhibitors have been shown to be effective in controlling Kaposi sarcoma in transplant patients, due to disruption of the antiapoptotic effect and angiogenesis dependent on the mTOR protein, which are essential for development and propagation of malignant cells. We present two case reports of patients with Kaposi sarcoma in lymph nodes and no skin lesions, who responded well to the immunosuppressive therapy switch with mTOR inhibitors.

Keywords: Kaposi sarcoma, human herpesvirus 8, kidney transplant, TOR serine-threonine kinases, lymph nodes.

\section{Introducción}

El trasplante renal constituye la mejor opción de tratamiento en un paciente con enfermedad renal avanzada, lo cual implica retos que buscan mejorar la supervivencia del injerto renal, y con ello, la implementación de nuevas terapias inmunosupresoras que se asocian con diferentes complicaciones, como son las infecciones y las neoplasias [1-3].

El cáncer en la actualidad afecta la expectativa y calidad de vida de los pacientes con trasplante de órgano sólido. Específicamente en los pacientes con trasplante de riñón, el riesgo alto de cáncer se atribuye a los virus oncogénicos, a la inmunosupresión y a una alteración de la inmunidad mediada por células T [1]. La incidencia de cáncer en los pacientes con trasplante renal se ha reportado entre 2 y 4 veces superior a la de la población general $[1,4,5]$, y representa la segunda causa de muerte después de la enfermedad cardiovascular $[1,6,7]$. Al compararse la incidencia de los diferentes tipos de cáncer entre pacientes con trasplante renal y el resto de la población, se ha observado que el sarcoma de Kaposi es hasta 300 veces más frecuente, los tumores no melanocíticos entre 2 y 40 veces, y el cáncer de labio hasta 10 veces $[1,2,8-10]$.
El sarcoma de Kaposi fue descrito por primera vez en 1872 por el dermatólogo húngaro Mortiz Kaposi [9]. Es causado por el virus herpes humano 8 (VHH-8) e inicialmente cobró importancia por su asociación con la infección por VIH y SIDA $[8,11,12]$; sin embargo, el sarcoma de Kaposi también se presenta en individuos sin infección por $\mathrm{VIH}$, al igual que en los receptores de trasplantes, y es endémico en África subsahariana [9]. En 1969 se demostró el primer caso asociado a la inmunosupresión en un paciente trasplantado renal $[13,14]$.

La seropositividad para el $\mathrm{VHH}-8$ varía enormemente a nivel mundial, siendo menor de $5 \%$ en Norteamérica, Japón y Europa, entre $5 \%$ a $20 \%$ en el Medio Oriente, entre $20 \%$ a $40 \%$ en Italia, y $>40 \%$ en África subsahariana y Amazonia [15-17]. Es de resaltar que no se ha encontrado correlación entre la incidencia del sarcoma de Kaposi y la prevalencia del virus; por ejemplo, en el Congo y en Perú hay una seroprevalencia para el $\mathrm{VHH}-8>70 \%$, en tanto que la tasa de incidencia de la enfermedad es solo entre 0,3 a 0,9 y <0,3 por 100.000 habitantes, respectivamente. En Colombia, por su parte, la seroprevalencia está entre $11 \%$ y $20 \%$, mientras que 
la tasa de incidencia de la enfermedad está entre 0,3 a 0,9 por 100.000 habitantes [8].

El VHH-8 puede infectar diferentes tipos de células, incluyendo las endoteliales, linfocitos $B$, células epiteliales, dendríticas, monocitos y fibroblastos. El diagnóstico de la infección se realiza a menudo con base en las características de las lesiones; sin embargo, debe ser confirmado por histología $[8,18]$. En años recientes, los inhibidores de mTOR, como everolimus y sirolimus, han mostrado ser efectivos en el control del sarcoma de Kaposi en los pacientes trasplantados, ya que se interrumpe el efecto antiapoptótico y la angiogénesis dependientes de la proteína mTOR, los cuales son esenciales para el desarrollo y la propagación de células malignas. Los inhibidores de mTOR también suprimen la traducción de mARNs que promueven la carcinogénesis $[19,20]$.

A continuación, se presentan dos casos de pacientes con sarcoma de Kaposi ganglionar, en nuestro centro de trasplante en Colombia, quienes respondieron de manera positiva al cambio del esquema inmunosupresor con inhibidores de mTOR.

\section{Caso clínico 1}

Hombre de 53 años con antecedente de insuficiencia renal de etiología desconocida, quien en marzo de 2011 inició con síntomas inespecíficos. Se realizaron paraclínicos que revelaron enfermedad renal avanzada. Dependiente de diálisis a partir de mayo de 2011 por 3 años, la cual posteriormente se cambió a hemodiálisis por disfunción de catéter. Presentó múltiples complicaciones con los accesos vasculares, incluyendo un síndrome de vena cava superior, con stent en la vena innominada izquierda y en la vena cava. El paciente recibió un trasplante renal de donante fallecido el 24 mayo de 2017. Incidencias operatorias: tiempo de isquemia fría (TIF) 14 horas, tiempo de isquemia caliente (TIC) 22 minutos; portador de catéter doble $\mathrm{J}$, el cual fue retirado 8 semanas posteriores al trasplante.

Para el esquema de inmunosupresión, se utilizó como terapia de inducción metilprednisolona y timoglobulina (ATG de conejo) $1 \mathrm{mg} / \mathrm{kg} /$ día por 3 días. Como terapia de mantenimiento se prescribió micofenolato de sodio $1.080 \mathrm{mg} /$ día, prednisolona $10 \mathrm{mg} /$ día y tacrolimus XL (liberación prolongada) $6 \mathrm{mg} /$ día. A los seis meses postrasplante, el paciente manifestó tos seca, pérdida de peso, deterioro de su clase funcional y disnea progresiva de medianos y pequeños esfuerzos. Se le realizó un ecocardiograma transtorácico que evidenció fracción de eyección del ventrículo izquierdo $60 \%$ y prueba de dobutamina $91 \%$ (negativa para isquemia), sin trastorno valvular. Por su parte, la PET-CT mostró múltiples adenopatías paravertebrales, prevasculares y subcarinales, con diámetros hasta de $35 \mathrm{~mm}$ (figura 1). En diciembre de 2017, el paciente es hospitalizado y sometido a mediastinoscopia con biopsia, con la cual se le diagnosticó sarcoma de Kaposi (figuras 2 y $\mathbf{3}$ ).

Un mes posterior al diagnóstico de sarcoma de Kaposi con compromiso de mediastino, y luego de conversión a esquema con prednisolona $5 \mathrm{mg} /$ día, everolimus $0,5 \mathrm{mg}$ cada 12 horas y micofenolato de sodio $360 \mathrm{mg}$ cada 12 horas, el paciente estaba asintomático y sin evidencia clínica ni paraclínica de actividad de la enfermedad, con niveles de everolimus de 6,2 ng/mL. En la figura 4 se observa la evolución de los paraclínicos. 


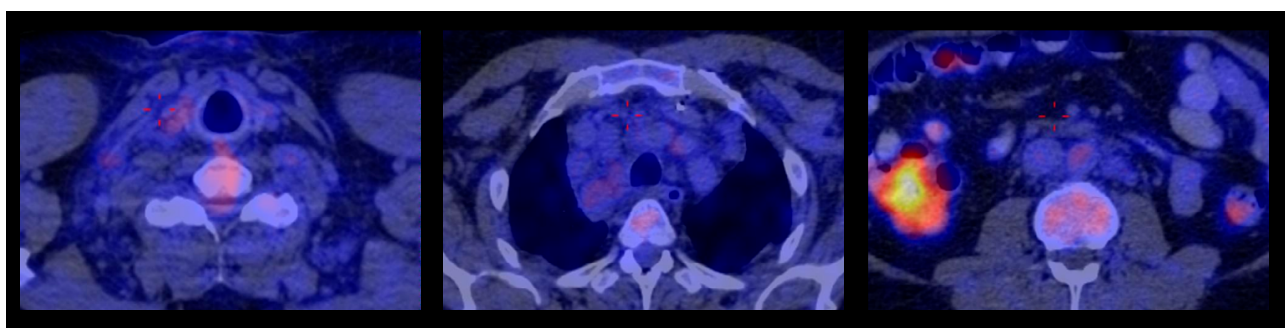

Figura 1. Imagen de PET-CT donde se observa aumento anormal del metabolismo con concentración leve de la FDG-F18 en la base del cuello bilateral en relación con varios ganglios linfáticos en el nivel $V$, de predominio izquierdo y menores de $10 \mathrm{~mm}$; en el mediastino en relación con varias adenopatías, las mayores de $25 \mathrm{~mm}$, localizadas entre la emergencia de los grandes vasos del cuello, espacio prevascular, región retrocavo pretraqueal, y región precarinal derecha; también, en ganglios linfáticos menores de $10 \mathrm{~mm}$ en el retroperitoneo en la región paraaórtica izquierda y retrocava.

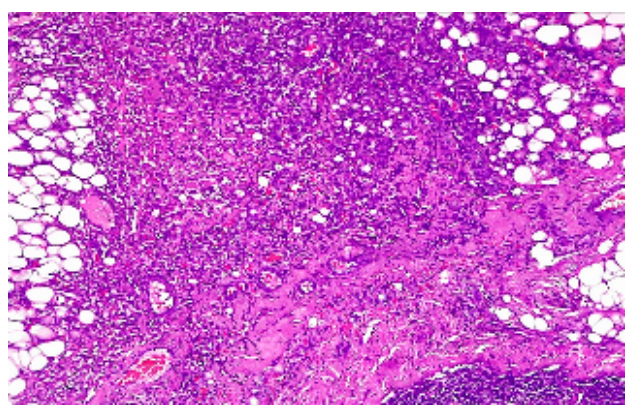

Figura 2. Ganglio linfático parcialmente reemplazado por una proliferación de canales vasculares de pequeño calibre, los cuales forman pequeñas hendiduras y se acompañan de extravasación de glóbulos rojos. Hay algunas células fusiformes con leve atipia que se disponen alrededor de los vasos. Hematoxilina y eosina (40x).

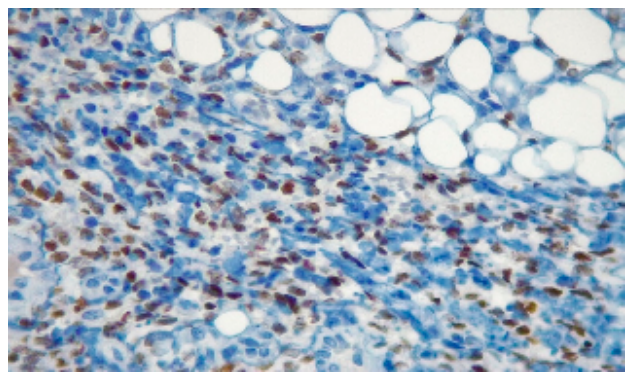

Figura 3. Prueba de inmunohistoquímica que muestra positividad nuclear, fuerte y difusa para virus herpes humano tipo $8(\mathrm{VHH}-$ 8) en las células neoplásicas (40x).

\section{Caso clínico 2}

Hombre de 28 años con antecedentes de enfermedad renal por aparente glomerulopatía. Refirió historia de proteinuria y biopsia renal con reporte desconocido, y estuvo en diálisis peritoneal los 2 años previos al momento del trasplante. Con hipertensión arterial desde el inicio de la enfermedad y trastorno de ansiedad en manejo por psiquiatría. Recibió trasplante renal de donante fallecido el 8 de enero de 2017. Incidencias operatorias: TIF 16 horas, TIC 20 minutos. Serología CMV donante/receptor +/+, compartía HLAA1, HLA-B1, HLA-DR. Terapia de inducción con metilprednisolona y timoglobulina $1 \mathrm{mg} / \mathrm{kg} /$ día por 3 días, y terapia de mantenimiento con micofenolato de sodio $1.080 \mathrm{mg} /$ día, prednisolona $10 \mathrm{mg} /$ día y tacrolimus XL $8 \mathrm{mg} /$ día.

El paciente presentó complicaciones postrasplante con ITU recurrente y pielonefritis del injerto renal. Función subóptima del injerto con creatinina basal de 1,6 mg/dL. En junio de 2018 tuvo pérdida no intencional de peso, deposiciones diarreicas esporádicas y aumento progresivo de adenopatías cervicales e inguinales, sin fiebre ni síntomas res- 
piratorios. En julio de 2018 fue hospitalizado para descartar infecciones oportunistas versus neoplasia postrasplante.
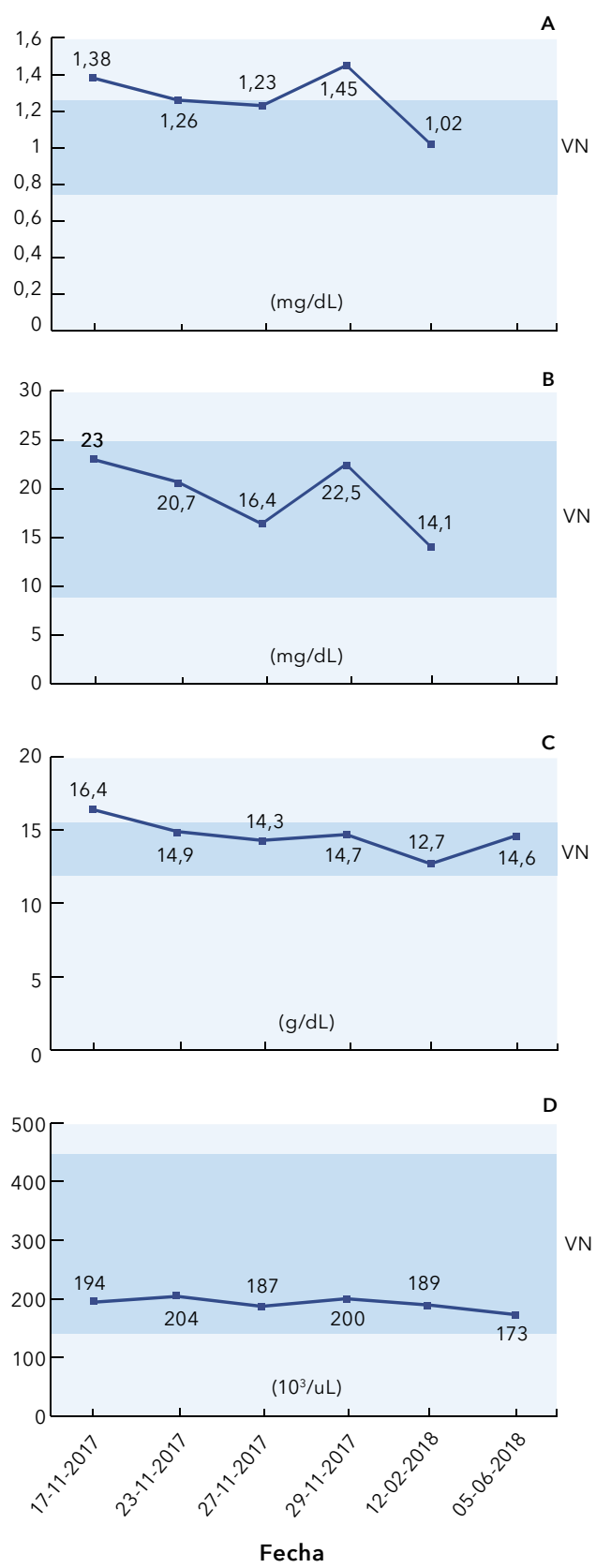

Figura 4. Evolución de los paraclínicos. (A) creatinina; (B) nitrógeno ureico; $(C)$ hemoglobina; (D) recuento de plaquetas. VN: rango de valores normales.
Se realizó diagnóstico de sarcoma de Kaposi con compromiso ganglionar (figuras 5, 6 y 7). Veinte días posteriores al diagnóstico, se hace conversión a esquema con prednisolona $5 \mathrm{mg} /$ día, everolimus $0,75 \mathrm{mg}$ cada 12 horas y micofenolato de sodio $360 \mathrm{mg}$ cada 12 horas. Se evidenciaron niveles de everolimus de $7,3 \mathrm{ng} / \mathrm{mL}$, con buena tolerancia a la inmunosupresión y disminución de más del $80 \%$ del tamaño de las adenopatías. Los paraclínicos y las imágenes solicitadas por oncología se observan en las figuras 8, 9 y 10.

\section{Discusión}

El sarcoma de Kaposi es una enfermedad rara en la población general, cuya incidencia puede estar entre 0,02\% a $0,07 \%[21,22]$. Su incidencia en el paciente con trasplante renal varía según la localización geográfica, siendo más frecuente en el Medio Oriente y en Arabia Saudita, en comparación con otras áreas del mundo como Estados Unidos y Australia [21]. Se ha descrito también

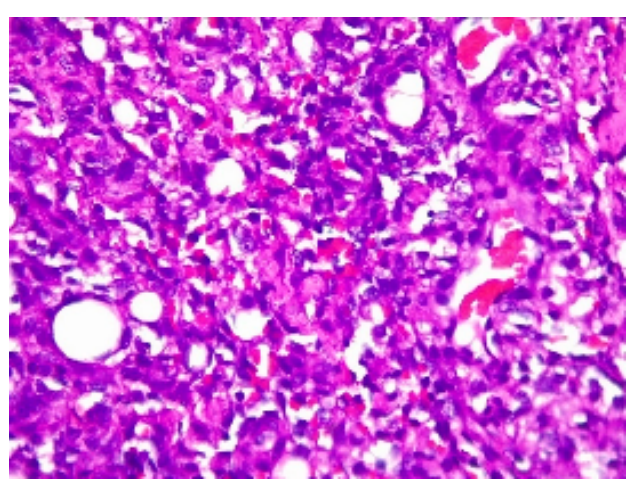

Figura 5. Biopsia de ganglio. Los cortes microscópicos muestran células fusiformes con citoplasma eosinófilo y núcleos elongados, exhibiendo atipia. Se observa gran extravasación de glóbulos rojos y proliferación vascular con células endoteliales prominentes. Hematoxilina y eosina (40x). 


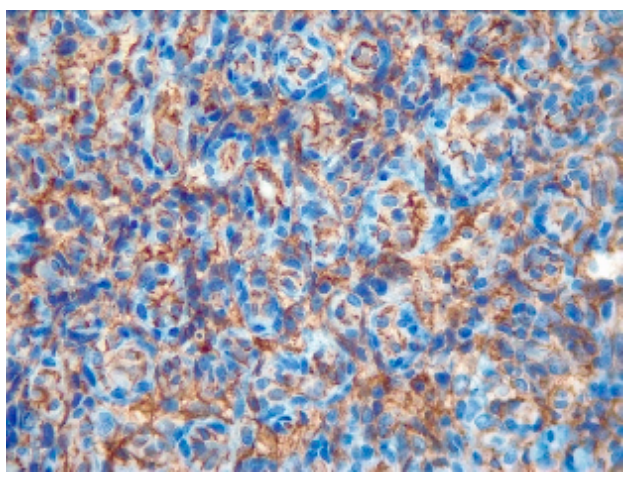

Figura 6. Prueba de inmunoperoxidasa para el marcador CD31, donde se puede evidenciar la abundante reactividad en la membrana celular de las células vasculares (40x).

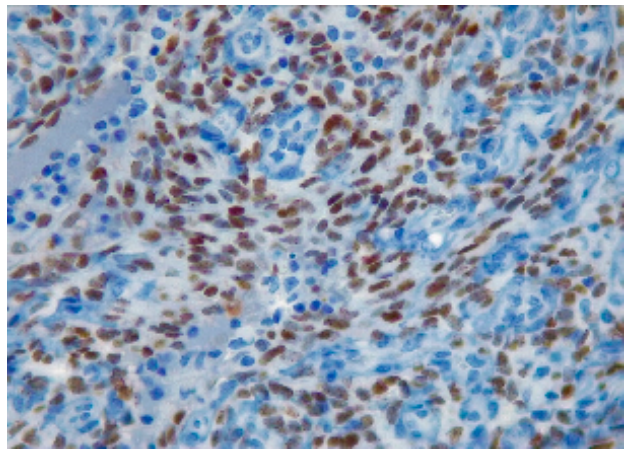

Figura 7. Prueba de inmunohistoquímica que muestra extensa positividad nuclear para virus herpes humano tipo $8(\mathrm{VHH}-8)$ en las células neoplásicas (40x).

asociación con la población judía y descendencia africana, e igualmente se describe un componente genético, siendo más frecuente en pacientes con HLA-DR5 y HLA-A2 [23], y en aquellos que reciben tratamiento con inhibidores de la calcineurina $[3,24,25]$.

Se conocen cuatro formas epidemiológicas del sarcoma de Kaposi. La forma clásica o esporádica, la cual se presenta en grupos étnicos con alta prevalencia del $\mathrm{VHH}-8$, que usualmente
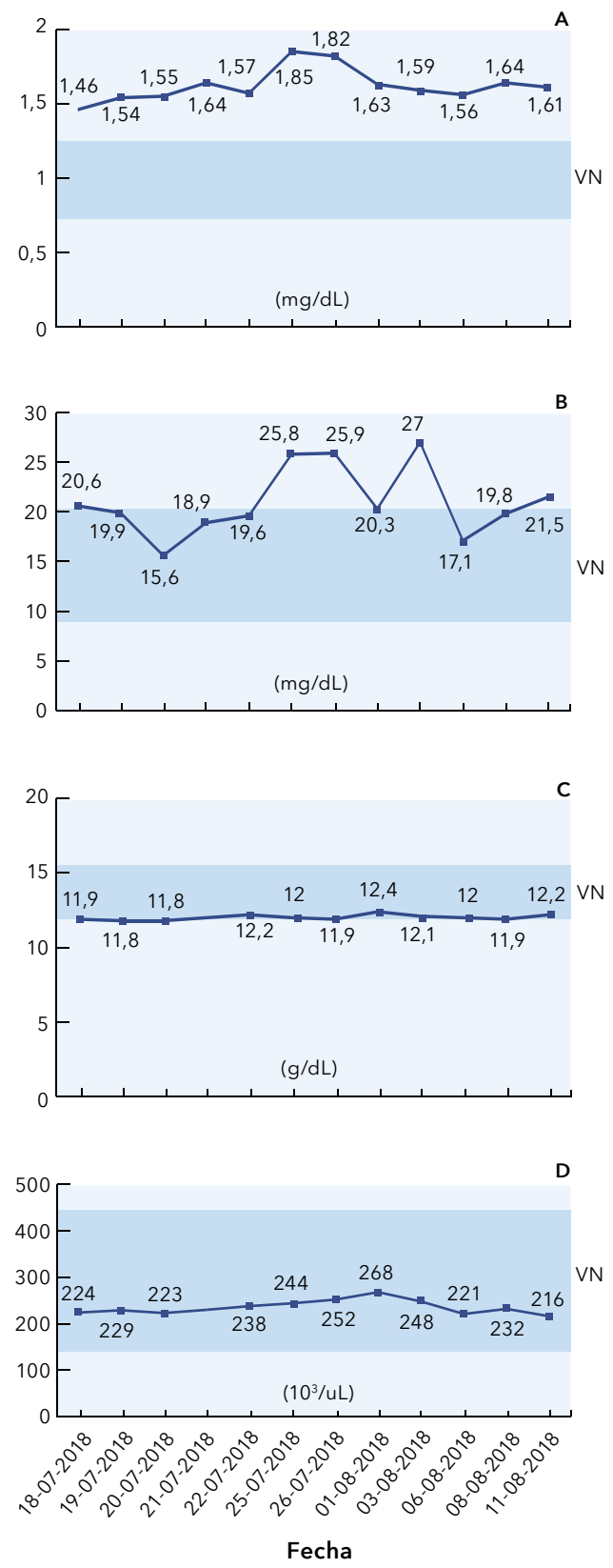

Figura 8. Evolución de los paraclínicos. (A) creatinina; (B) nitrógeno ureico; (C) hemoglobina; (D) recuento de plaquetas. VN: rango de valores normales.

se manifiesta como unas pocas lesiones en las extremidades inferiores. La forma epidémica o asociada al SIDA, 
en la cual hay aparición de múltiples lesiones cutáneas en las extremidades, tronco y rostro, con compromiso mucoso y visceral en el $15 \%$ a $20 \%$. La forma endémica, en África subsahariana, debido a la trasmisión del virus por

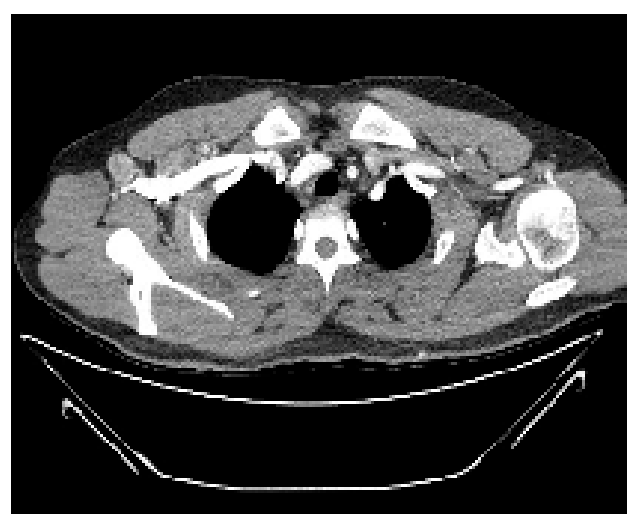

Figura 9. Tomografía de cuello y tórax donde se observan múltiples adenopatías. Cervicales en las estaciones ganglionares: adenopatía nivel $V$ derecha $14 \times 20 \mathrm{~mm}$ y nivel $\mathrm{V}$ izquierda $12 \times 15 \mathrm{~mm}$. Mediastinales: paratraqueal inferior derecha $25 \times 27$ $\mathrm{mm}$ e inferior izquierda $15 \times 24 \mathrm{~mm}$, parahiliar derecha $12 \times 18 \mathrm{~mm}$ e izquierda $17 \times 22$ $\mathrm{mm}$; también, axilar derecha $24 \times 17 \mathrm{~mm}$ e izquierda $22 \times 33 \mathrm{~mm}$.

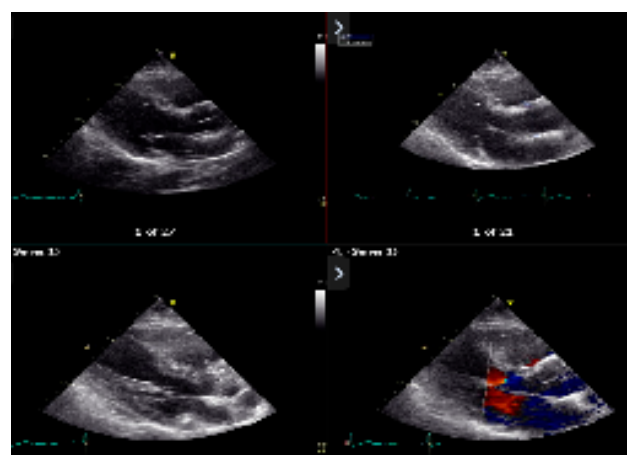

Figura 10. Ecocardiograma con fracción de eyección del ventrículo izquierdo (FEVI) $65 \pm 5 \%$. Ventrículos izquierdo y derecho con morfología normal. Presión sistólica de arteria pulmonar $37 \mathrm{mmHg}$. Sin trastorno valvular y contractilidad normal. saliva durante la infancia, en la cual los niños presentan adenopatías múltiples con linfedema y diseminación visceral, mientras que los adultos presentan la clínica similar a la forma clásica o esporádica. Por último, la forma iatrogénica, como la que se observa en los pacientes trasplantados, se correlaciona con el grado de inmunosupresión e incompatibilidad HLA, y se manifiesta con mayor frecuencia como lesiones cutáneas, pero puede también ocurrir compromiso mucoso y visceral [8]. Es de anotar que ninguno de los dos casos expuestos presentó lesiones en piel.

El $\mathrm{VHH}-8$ es el agente etiológico del sarcoma de Kaposi y de otras enfermedades linfoproliferativas de células $B$. Es un virus ADN que pertenece a la familia de los Herpesviridae y se conoce también con el nombre de virus herpes asociado a sarcoma de Kaposi. Se transmite a través de los líquidos corporales, principalmente la saliva, y la mayoría de las infecciones son subclínicas [18]. Una vez el virus infecta la célula, su genoma es transportado al núcleo donde permanece como un episoma y entra en estado de latencia, con reactivaciones esporádicas durante su ciclo de vida. En presencia de estímulos, como la inmunosupresión a la que son sometidos los pacientes trasplantados, el virus se reactiva e inicia la desregulación de varias vías de señalización que garantizan la proliferación y supervivencia de las células, promoviendo la oncogénesis, además de inducir la replicación viral, lo cual a su vez aumenta la secreción de citoquinas y factores de crecimiento que inducen angiogénesis e inflamación, contribuyendo a la patogénesis causada por el virus [8].

Como se mencionó, estudios previos han mostrado que la incidencia del sarcoma de Kaposi aumenta sustancialmente en los pacientes sometidos a 
trasplantes de órganos. Este aumento se observa particularmente en los receptores con serología positiva para VHH-8 previa al trasplante, lo cual sugiere que la reactivación del virus asociada a la terapia inmunosupresora, es el factor desencadenante, [26,27]. Por lo general, la enfermedad se presenta en el primer año postrasplante, pero puede aparecer desde pocas semanas hasta muchos años después. En estos pacientes se ha observado que el sarcoma de Kaposi se asocia con una edad más avanzada, con episodios de rechazo agudo y con tratamientos con esteroides y azatioprina, entre otros factores $[28,29]$. Por el contrario, los esquemas de inmunosupresión con inhibidores de mTOR han demostrado protección y control de los diferentes tipos de neoplasias, incluido el sarcoma de Kaposi [1,2,15,30,31], lo cual se reportó recientemente en un metaanálisis, sin observarse un aumento en la mortalidad ni en la pérdida del injerto [32], similar a lo sucedido con ambos pacientes presentados en los casos, con óptimos resultados.

Las manifestaciones del sarcoma de Kaposi en el paciente trasplantado se caracterizan por lesiones en piel, principalmente en extremidades inferiores, de características angiomatosas, violáceas, no pruriginosas, a menudo asociadas a compromiso orofaríngeo y conjuntival, y con menor frecuencia a compromiso visceral, siendo el aparato gastrointestinal, los pulmones y los ganglios linfáticos los sitios más afectados; solo el $10 \%$ de los casos tiene presentación visceral exclusiva [21]. La enfermedad es más frecuente en mayores de 60 años [33], contrario a los casos expuestos, cuyas edades eran de 28 y 53 años. En ambos pacientes hubo presentación visceral y las manifestaciones tuvieron su aparición en menos de un año postrasplante, con una bue- na respuesta en cuanto al manejo terapéutico, que incluyó reducción de la inmunosupresión y el uso de inhibidores de mTOR, logrando así la disminución progresiva de las lesiones.

El diagnóstico histológico se puede realizar usualmente mediante la coloración con hematoxilina y eosina. Entre los hallazgos se encuentran proliferación vascular, extravasación de glóbulos rojos y células fusiformes [8], como los encontrados en ambos pacientes (figuras 2 y 5). Adicionalmente, puede haber acumulación de hemosiderina e infiltrado inflamatorio. Las técnicas de inmunohistoquímica/inmunoperoxidasa también pueden contribuir al diagnóstico, con anticuerpos específicos que detecten la presencia de marcadores como CD31 (figura 6) o que detecten proteínas virales (figuras $\mathbf{3}$ y 7); estas técnicas también ayudaron a confirmar el diagnóstico en los casos presentados $[8,9,34]$.

\section{Conclusión}

La incidencia del sarcoma de Kaposi es mayor en los pacientes trasplantados debido a los esquemas de inmunosupresión. En los receptores de trasplante renal, la intensidad y duración de la inmunosupresión, y la seropositividad para el $\mathrm{VHH}-8$ pretrasplante, aumentan el riesgo de desarrollar la enfermedad. La disminución de la inmunosupresión, junto con la conversión de los inhibidores de calcineurinas por inhibidores de mTOR pueden contribuir al control de la enfermedad en los estadios tempranos de la enfermedad, como ocurrió con ambos casos presentados.

En Colombia no hay estudios sobre la prevalencia del sarcoma de Kaposi, de ahí la importancia de conocer las características clínicas y epidemiológicas, 
Sarcoma de Kaposi ganglionar postrasplante renal: reporte de dos casos en un centro de trasplante colombiano

que permitan cada vez obtener mejores resultados a corto y a largo plazo. Aún se requieren más estudios para evaluar el esquema inmunosupresor óptimo e identificar a aquellos pacientes que requieran dosis más bajas, con el fin de lograrse un punto de equilibrio que permita evitar la pérdida del injerto y disminuir el riesgo de complicaciones derivadas de la inmunosupresión, como sucede con el sarcoma de Kaposi.

\section{Referencias}

1. Au E, Wong G, Chapman JR. Cancer in kidney transplant recipients. Nat Rev Nephrol 2018;14:508-520. https://doi.org/10.1038/ s41581-018-0022-6.

2. Cheung CY, Tang SCW. An update on cancer after kidney transplantation. Nephrol Dial Transplant 2019;34:914-920. https://doi.org/10.1093/ ndt/gfy262.

3. Campistol JM, Eris J, Oberbauer R, Friend $\mathbf{P}$, Hutchison B, Morales JM, et al. Sirolimus therapy after early cyclosporine withdrawal reduces the risk for cancer in adult renal transplantation. J Am Soc Nephrol 2006;17:581-589. https://doi.org/10.1681/asn.2005090993.

4. Gutiérrez-Dalmau A, Revuelta I, Campistol JM. Renal transplantation and cancer: Focus on immunosuppressive therapy. Trends in Transplant 2007;1:3-14.

5. Campistol JM, Cuervas-Mons V, Manito N, Almenar L, Arias M, Casafont F, et al. New concepts and best practices for management of pre- and post-transplantation cancer. Transplant Rev (Orlando) 2012;26:261-279. https:// doi.org/10.1016/j.trre.2012.07.001.

6. Siegel R, Naishadham D, Jemal A. Cancer statistics, 2012. CA Cancer J Clin 2012;62:10-29. https://doi.org/10.3322/caac.20138.

7. Webster A, Wong G, McDonald S. Australia and New Zealand dialysis and transplant registry. The 32th annual report. Chapter 10, Cancer report. Adelaide, South Australia: South Australian Health and Medical Research Institute (SAHMRI); 2009. Acceso 05 de noviembre de 2020. Disponible en http://www.anzdata.org.au/.
8. Cesarman E, Damania B, Krown SE, Martin J, Bower M, Whitby D. Kaposi sarcoma. Nat Rev Dis Primers 2019;5:9. https://doi.org/10.1038/ s41572-019-0060-9.

9. Bishop BN, Lynch DT. Kaposi sarcoma. Treasure Island (FL): StatPearls Publishing; 2021. Acceso 14 de noviembre de 2020. Disponible en https:// www.ncbi.nlm.nih.gov/books/NBK534839/.

10. Kasiske BL, Snyder JJ, Gilbertson DT, Wang C. Cancer after kidney transplantation in the United States. Am J Transplant 2004;4:905-913. https:// doi.org/10.1111/j.1600-6143.2004.00450.x.

11. Plancoulaine S, Gessain A. [Epidemiological aspects of human herpesvirus 8 infection and of Kaposi's sarcoma]. Med Mal Infect 2005;35:314-321. https://doi.org/10.1016/j. medmal.2005.02.009

12. Volberding PA. 400 - Hematology and oncology in patients with human immunodeficiency virus infection. In: Goldman L, Schafer Al, eds. Goldman's Cecil Medicine (Twenty Fourth Edition). Philadelphia: W.B. Saunders; 2012. p. 2212-2217.

13. Gutiérrez-Dalmau A, Campistol JM. Kaposi's sarcoma after renal transplantation. $N$ Engl J Med 2005;353:846-847.

14. Schulte $B$, Linke D, Klumpp $S$, Schaller $M$, Riess T, Autenrieth IB, et al. Bartonella quintana variably expressed outer membrane proteins mediate vascular endothelial growth factor secretion but not host cell adherence. Infect Immun 2006;74:5003-5013. https://doi. org/10.1128/iai.00663-06.

15. Piselli $\mathbf{P}$, Taborelli M, Cimaglia C, Serraino D. Decreased incidence of Kaposi sarcoma after kidney transplant in Italy and role of mTOR-inhibitors: 1997-2016. Int J Cancer 2019;145:597598. https://doi.org/10.1002/ijc.32098.

16. Ahmadpoor P. Human herpesvirus- 8 and Kaposi sarcoma after kidney transplantation: mechanisms of tumor genesis. Iran J Kidney Dis 2009;3:121-126.

17. Kalt I, Masa SR, Sarid R. Linking the Kaposi's sarcoma-associated herpesvirus (KSHV/HHV8 ) to human malignancies. Methods Mol Biol 2009;471:387-407. https://doi.org/10.1007/9781-59745-416-2_19.

18. Rewane A, Tadi P. Herpes virus type 8. Treasure Island (FL): StatPearls Publishing; 2021. Acceso 
1 de noviembre de 2020. Disponible en https:// www.ncbi.nlm.nih.gov/books/NBK556023/.

19. Holdaas $H$, De Simone $P$, Zuckermann A. Everolimus and malignancy after solid organ transplantation: A clinical update. J Transplant 2016;2016:4369574. https://doi. org/10.1155/2016/4369574.

20. Detroyer D, Deraedt $K$, Schöffski $P$, Hauben E, Lagrou K, Naesens M, et al. Resolution of diffuse skin and systemic Kaposi's sarcoma in a renal transplant recipient after introduction of everolimus: a case report. Transpl Infect Dis 2015;17:303-307. https://doi.org/https://doi. org/10.1111/tid.12357.

21. Einollahi B. Kaposi sarcoma after kidney transplantation. Iran J Kidney Dis 2007;1:2-11.

22. Antman $K$, Chang $\mathbf{Y}$. Kaposi's sarcoma. N Engl J Med 2000;342:1027-1038. https://doi. org/10.1056/nejm200004063421407.

23. García PK, Córdoba Buritica JP, Rodríguez MP, Echeverri JE, Lozano E, Benavides C. Sarcoma de Kaposi en trasplante renal, respuesta a sirolimus: reporte de caso y revisión de la literatura científica. Univer Med 2010;52:209-218.

24. Moray G, Başaran O, Yağmurdur MC, Emiroğlu R, Bilgin N, Haberal M. Immunosuppressive therapy and Kaposi's sarcoma after kidney transplantation. Transplant Proc 2004;36:168-170. https://doi.org/10.1016/j. transproceed.2003.11.016.

25. Penn I. Cancers in cyclosporine-treated vs azathioprine-treated patients. Transplant Proc 1996;28:876-878.

26. Fang $Q$, Wang $X$, Liu $Z$, Zhu M, Ding $M$, Minhas $\mathbf{V}$, et al. Seroprevalence of human herpesvirus 8 and its impact on the hemoglobin level in patients of end stage of renal diseases. J Med Virol 2018;90:338-343. https://doi. org/10.1002/jmv.24937.

27. Francès $C$, Marcelin AG, Legendre $C$, Chevret $\mathbf{S}$, Dussaix $\mathbf{E}$, Lejeune J, et al. The im- pact of preexisting or acquired Kaposi sarcoma herpesvirus infection in kidney transplant recipients on morbidity and survival. Am J Transplant 2009;9:2580-2586. https://doi. org/10.1111/j.1600-6143.2009.02816.x.

28. Cahoon EK, Linet MS, Clarke CA, Pawlish KS, Engels EA, Pfeiffer RM. Risk of Kaposi sarcoma after solid organ transplantation in the United States. Int J Cancer 2018;143:2741-2748. https://doi.org/10.1002/ijc.31735.

29. Donia AF, Fouda MA, Ghoneim ME, Refaie AF, Ali-El-Dein B. The previously common post-kidney transplant Kaposi sarcoma has become non-existent for a decade: an Egyptian experience. J Cancer Res Clin Oncol 2020. https://doi.org/10.1007/s00432-020-03433-1.

30. Campistol JM, Gutierrez-Dalmau A, Torregrosa JV. Conversion to sirolimus: a successful treatment for posttransplantation Kaposi's sarcoma. Transplantation 2004;77:760-762. https://doi.org/10.1097/01. tp.0000115344.18025.0b.

31. Stallone G, Schena A, Infante B, Di Paolo S, Loverre A, Maggio G, et al. Sirolimus for Kaposi's sarcoma in renal-transplant recipients. N Engl J Med 2005;352:1317-1323. https://doi. org/10.1056/NEJMoa042831.

32. Wolf $S$, Hoffmann VS, Habicht A, Kauke T, Bucher J, Schoenberg $\mathbf{M}$, et al. Effects of mTOR-ls on malignancy and survival following renal transplantation: A systematic review and meta-analysis of randomized trials with a minimum followup of 24 months. PLoS One 2018;13:e0194975. https://doi.org/10.1371/journal.pone.0194975.

33. Abbaszadeh S, Taheri S. Kaposi's sarcoma after renal transplantation. Saudi J Kidney Dis Transpl 2009;20:775-778.

34. Nauman A, Arshad A, Mujeeb I, Asim M. Renal transplant failure due to infiltrating Kaposi sarcoma. Exp Clin Transplant 2019;17:554-557. https://doi.org/10.6002/ect.2017.0052. 